\title{
Temor y angustia: experiencia del paciente en la unidad de cuidados intensivos
}

\author{
Fear and anguish: patient experience in intensive care unit \\ Medo e angústia: experiência do paciente na unidade de terapia intensiva \\ Keydis Sulay Ruidiaz-Gómez ${ }^{l}$ \\ Shirley Fernández-Aragón ${ }^{2}$
}

\section{Resumen}

Objetivo: Comprender las experiencias de pacientes egresados de la unidad de cuidado intensivo de una institución clínica de tercer nivel de atención de la Ciudad de Cartagena utilizando como enfoque la teoría del desarrollo humano de Rosemarie Rizzo Parse. Materiales y métodos: Estudio fenomenológico - interpretativo con perspectiva Hermenéutica, participaron 11 adultos egresados de la unidad de cuidados intensivos, se utilizó la técnica de entrevista a profundidad con una duración de 30 y 40 minutos, los datos se analizaron a través de la técnica de intencionalidad, reducción y constitución. Resultados: Los participantes del estudio fueron ocho hombres y tres mujeres adultos, su experiencia la describieron con sentimientos de temor y angustia que incluyen los subtemas: Buen trato; imaginación de muerte: Mi vida, el trabajo y la familia; Sentimientos de soledad; Fortaleza espiritual y Cambios de vida. Conclusión: Estar hospitalizado en la unidad de cuidados intensivos logró desencadenar una experiencia descrita como "temor y angustia", para los participantes es primordial el núcleo familiar, la fortaleza espiritual, la transformación y cambios de vida.

Palabras clave: Unidades de cuidados intensivos; experiencias de vida; hospitalización; enfermería, teoría de enfermería.

\section{Abstract}

Objective: To understand the experiences of patients graduated from an intensive care unit of a clinical institution of the third level of care of the City of Cartagena using Rosemarie Rizzo Parse's theory of human development as a focus. Materials and methods: Phenomenological study- interpretive with Hermeneutic perspective, involved 11 adults graduated from the intensive care unit participated, the in-depth interview technique was used with a duration of 30 and 40 minutes, the data were analyzed through the technique of intentionality, reduction and constitution. Results: Study participants were eight men and three adult women, their experience describing it with feelings of fear and anguish supported by good treatment, Imagination of death, my life, work and family,

\section{Autor de correspondencia}

${ }^{1 *}$ Enfermera, Magister en Enfermería. Docente Universidad del Sinú EBZ, Cartagena. Estudiante Doctorado en enfermería Universidad de Antioquia. Cartagena, Colombia. Correo: coordinvestenfermeria@unisinucartagena. edu.co. . (iD)

${ }^{2}$ Enfermera, Magister en enfermería, Docente Corporación Universitaria Rafael Núñez. Cartagena, Colombia. Correo: shirley.fernandez@,curnvirtual.edu.co. (iD)

Recibido: 25 noviembre 2019. Aprobado: 6 abril 2020 .

Para citar este artículo:

Ruidiaz-Gómez KS, Fernández-Aragón S. Temor y angustia: experiencia del paciente en la unidad de cuidados intensivos. Rev. cienc. cuidad. 2020; 17(3):7-19. https://doi.org/10.22463/17949831.1695

C Universidad Francisco de Paula Santander. Este es un artículo bajo la licencia CC-BYNC-ND

(c) (1) (3) $\Theta$ 
Feelings of solitude, spiritual strength and life changes. Conclusion: being hospitalized in the intensive care unit succeeded in triggering an experience described as "fear and distress", the family nucleus, spiritual strength and the transformation in life changes are paramount for participants.

Keywords: Intensive Care Units; Life experiences; Hospitalization; Nursing; Nursing Theory.

\section{Resumo}

Objetivo: Entender as experiências de pacientes formados na unidade de terapia intensiva de uma instituição clínica de terceiro nível na cidade de Cartagena utilizando como foco a teoria do desenvolvimento humano de Rosemarie Rizzo Parse. Materiais e métodos: Estudo fenomenológico - interpretativo com uma perspectiva hermenêutica, participaram 11 adultos formados na unidade de terapia intensiva, foi utilizada a técnica de entrevista em profundidade com duração de 30 e 40 minutos, os dados foram analisados através da técnica de intencionalidade, redução e constituição. Resultados: Os participantes do estudo foram oito homens adultos e três mulheres adultas. Eles descreveram suas experiências com sentimentos de medo e angústia que incluíram os subtópicos: Bom Tratamento; Imaginação da Morte: Minha Vida, Trabalho e Família; Sentimentos de Solidão; Força Espiritual e Mudanças de Vida. Conclusão: Ser hospitalizado na unidade de terapia intensiva conseguiu desencadear uma experiência descrita como "medo e angústia". Para os participantes, a unidade familiar, a força espiritual, a transformação e as mudanças de vida são primordiais.

Palavras-chave: Unidades de Terapia Intensiva; Hospitalização; Enfermagem, Teoria de Enfermagem.

\section{Introducción}

Estar hospitalizado en una unidad de cuidados intensivos (UCI) genera alteraciones emocionales en el individuo como ansiedad, estrés, angustia, depresión o el denominado síndrome de cuidados intensivos, este se presenta por factores como el ruido excesivo que dificulta el sueño, la luz que limita descanso y las diversas medidas empleadas para la ventilación que impiden la comunicación en los enfermos (1-3).

Diversos autores se han dedicado al estudio de esta problemática, con el fin de conocer las experiencias y sentimientos presentes en los pacientes hospitalizados en la unidad de cuidados intensivos, Amorós et al. (4), y Beltrán (5-6). Describen las experiencias del paciente teniendo en cuenta que la UCI es un entorno impersonal y tecnológicamente invasivo, su estructura arquitectónica favorece la pérdida de la intimidad, cambios en la relación profesional-paciente donde la tecnología parece certificar el discurso de este, convirtiendo el hecho en una experiencia negativa; además, provoca sufrimiento en el individuo por el tratamiento y los procedimientos realizados para resolver la enfermedad (5-7).

La experiencia de estar hospitalizado en una UCI es una situación difícil y dolorosa para los pacientes. Esto genera sentimientos negativos en la persona, que marcan significativamente su experiencia de vida, pero que también desencadenan aspectos positivos derivados de la atención humanizada que brinda el personal de enfermería y la experiencia renovadora que genera un cambio significativo para la vida del paciente, al considerar que es una nueva oportunidad para volver a vivir y para un nuevo comienzo (8-9).

En concordancia con la premisa anterior, enfermería históricamente ha pasado de una etapa tradicional de cuidado a una etapa de transformación (10) donde da valor y sentido a las experiencias vividas por el enfermo, su entorno y familia. $\mathrm{La}$ constante interacción del individuo con el entorno, lleva a los enfermeros a enlazar diferentes dimensiones (sociales, individuales) que influyen en el cuidado profesional, para obtener elementos sólidos que ayudan a pensar al ser humano en toda su dimensión holística, además de crear una nueva visión ética encaminada a reducir en lo posible el miedo, el dolor y el sufrimiento causado 
por esa experiencia (10-11).

A lo largo de la historia, la transformación paradigmática de la enfermería como disciplina ha sido lenta y compleja. Por tanto, se toma como referente la teoría del desarrollo humano propuesta por Rosemarie Rizzo Parse (12), porque permite estructurar los significados del ser humano inmerso en una situación, configurar los patrones rítmicos y co-trascendentes de las situaciones discontinuas del ser humano, en este caso, la experiencia de estar hospitalizado en una unidad de cuidados intensivos. El pensamiento del desarrollo humano articulado con el pensamiento existencialista fenomenológico da las bases para un sistema conceptual que permite comprender las experiencias vividas, la libertad y su participación en situaciones de vida, propia del enfoque hermenéutico,. Así mismo, permite explorar la esencia de las experiencias vividas para conseguir una comprensión más profunda de los fenómenos humanos universales.

En Cartagena son pocas las investigaciones realizadas sobre este enfoque individualista, sus aportes se han orientado al afrontamiento y adaptación de la enfermedad, por ende, una investigación de este tipo muestra una visión hermenéutica- transformadora de cuidado, que lleva al profesional de enfermería a utilizar ideas innovadoras, focalizadas en los fenómenos universales de la salud y los diferentes momentos de vida, lo cual, contribuirá al mejoramiento de la práctica de enfermería.

\section{Objetivo}

\section{Objetivo General}

Comprender las experiencias de los pacientes adultos egresados de la unidad de cuidado intensivo de una institución clínica de la Ciudad de Cartagena, utilizando como enfoque la teoría del desarrollo humano de Rizzo Parse.

\section{Materiales y métodos}

Se realizó un estudio fenomenológico - interpretativo con perspectiva hermenéutica según Heidegger (13-15). La investigación se desarrolló con la participación de 11 adultos que egresaron de la UCI, que al momento de la entrevista se encontraban en las salas de hospitalización; quienes participaron en el estudio de forma voluntaria y sin remuneración económica o de otro tipo por parte de los investigadores. Con cada participante se realizó un acercamiento y se estableció una comunicación directa para dar a conocer los objetivos y finalidades del estudio.

Esta investigación implementó una técnica de muestreo intencional (15), mediada por los criterios de inclusión: ser paciente con rango de edad entre 30 y 80 años (población frecuente de hospitalización), y haber estado hospitalizado en la UCI por más de 72 horas.

Para la recolección de la información se utilizó la técnica de entrevista a profundidad (16), con el fin de conocer la experiencia vivida en sentido fenomenológico, las dimensiones de la vida personal, las experiencias significativas la relación con las experiencias en torno al tema de interés; para de este modo, poder buscar relaciones entre situaciones de globalidad y cada experiencia en su particularidad.

La entrevista comprendió en su primera parte los datos demográficos del participante, luego, de manera introductoria se inició la entrevista con la siguiente pregunta orientadora: ¿cuál fue la experiencia vivida mientras estuvo en la UCI? Las entrevistas fueron grabadas en medio magnético (grabadora de periodismo) y transcritas en su totalidad en un procesador de texto por el mismo investigador tan pronto se realizaba la entrevista, con el objeto de asegurar la información, tal como era expresada por el entrevistado (17). Cada entrevista estuvo identificada con un código y enumeradas de manera secuencial. Las entrevistas tuvieron una duración entre 30 y 40 minutos aproximadamente, a cada participante se le realizó un total de 2 a 3 entrevistas con el fin de profundizar en las experiencias significativas.

Una vez recolectados los datos de la entrevista, se analizaron según la perspectiva Heideggeriana, el proceso contempla tres etapas: la intencionalidad, la reducción y la constitución. La primera, consiste en una vez terminadas las entrevistas se realiza la transcripción las cuales se confrontan mediante la lectura del texto y la escucha del audio para dar confirmación de los datos digitados $y$ de esta manera hacer un detallado general de toda la información recolectada (13). La segunda, implica la toma de decisiones por parte de los investigadores respecto a lo que es principal o relevante y lo que no es. Se realiza una sustracción en las transcripciones de las entrevistas, con el fin de excluir aquellas desviaciones y cambios bruscos en los temas y expresiones $(16,18)$; por último, una vez terminado el proceso de recolección de la información, se realizó una reorganización de los datos que permitió establecer conexiones entre los principios de la teoría del desarrollo humano y los fenómenos para finalmente reagrupar 


\section{Keydis Sulay Ruidiaz-Gómez, Shirley Fernández-Aragón.}

los datos (19).

ISSN-PRINT

1794-9831

E-ISSN 2322-7028

Vol. 17 No. 3

Sep - Dic 2020

Cúcuta, Colombia
Para testificar la rigurosidad y calidad del estudio se tuvo en cuenta los criterios de rigor de la investigación cualitativa según Lincoln y Guba (20-22), credibilidad, confirmabilidad, aplicabilidad y auditabilidad, para dar cuenta de ello se contactó a cada participante para realizar lectura de las entrevistas iniciales, se comparó de manera constante las grabaciones de audio y los escritos de las entrevistas con hallazgos contrastados en la literatura, las limitaciones y alcance de la investigación, al finalizar del estudio se socializaron las conclusiones finales a los participantes y personal de la institución clínica. Además, se utilizó la herramienta de análisis cualitativo CASPe (23) para obtener un análisis crítico de la perspectiva teórica e interna del estudio según el rigor, la relevancia y credibilidad. También, se validaron los datos a través de la triangulación de los hallazgos con la literatura, las entrevistas, dos expertos en el tema y el propio investigador.

Previo a la entrevista se contó con la autorización escrita y verbal de los participantes; las entrevistas, conversaciones informales y notas en audio, trascripciones y análisis, fueron almacenadas bajo códigos de seguridad en el programa de Gmail de Drop Box con el fin de proteger el anonimato de los participantes. Para el manejo de los datos se emplearon seudónimos y códigos en cada una de las entrevistas con el objeto de salvaguardar la identidad de cada participante.

Referente a los aspectos éticos se atendieron los criterios de la resolución 008430 de 1993 (24), la cual catalogó la investigación como de riesgo mínimo, así como también los siete principios éticos de Ezekiel Emanuel (25) y los principios bioéticos de beneficencia y no maleficencia. El trabajo fue sometido a la aprobación del Comité de Investigaciones del programa de enfermería y al Comité de Ética y Bioética de la institución bajo el código de proyecto ENF-PD 2018-05.

\section{Resultados}

Los participantes del estudio fueron ocho hombres y tres mujeres adultos, con rango de edades entre los 32 y 74 años, con bachillerato completo e incompleto y formación técnica y profesional, variadas ocupaciones, en su mayoría casados, de religión católica diferentes estratos socioeconómicos; con hospitalización en UCI de 5 a 45 días. Entre las patologías que presentaron los participantes para el ingreso a la unidad, se encontraron falla cardiaca, dolor torácico, infarto agudo de miocardio, trauma cráneo encefálico, hemorra- gias de vías digestivas entre otras.

La experiencia se aprecia desde los pacientes egresados de una unidad de cuidados intensivos de Cartagena, donde a la luz de los principios de la teoría del desarrollo humano propuesta por $\mathrm{Ri}$ zzo (12) se establece el ser humano como ser que constituye su experiencia de vida inmerso en un entorno intencionado donde reconoce su mundo, actúa frente a las adversidades y le da significado a las situaciones convirtiéndose en un ser indivisible.

Los hallazgos mostraron un tema central donde se condensan las mayores experiencias individuales y seis subtemas relacionados con los principios de la Teoría del Desarrollo Humano (Figura 1). A continuación se presentan los temas que surgen de la experiencia:

\section{Tema central: Temor y Angustia}

El temor y la angustia fueron los sentimientos expresados por los participantes en el tiempo que estuvieron hospitalizados en la UCI, el paciente genera ideas y creencias a raíz de la situación que enfrenta. Al ingresar a la unidad creen que algo no está bien en su salud, por lo que ven la muerte muy cercana, desconocen su propia enfermedad y todo lo que ocurre alrededor de ellos.

La hospitalización en la unidad de cuidados intensivos para los participantes se convirtió en un fenómeno desconocido en todos los ámbitos (personal, familiar y social), dicha experiencia trajo consigo vivencias inesperadas, en ese contexto los pacientes se convierten en seres más vulnerables y sensibles ante su situación.

Rizzo (12) establece que el conocimiento vivido va acorde a los ritmos expresados por los patrones sociales de vida, aquí el individuo, en este caso, los adultos egresados de la UCI, crean su propia experiencia a través de la incertidumbre, la tristeza y la falta de libertad, por eso, a pesar de tener un equipo interdisciplinario al cuidado de su salud, la unidad de cuidados intensivos les representa un espacio donde experimentan sentimientos inesperados que generan situaciones desesperantes, temerosas y no deseadas, expresado así:
PE2:"Bueno...., esa experiencia no se la deseo a nadie" $[\ldots .$.

PE5: “me generaba mucho temor, mucho miedo, sentía angustia. sinceramente... mucho, mucho miedo, mucho temor".

PE8: “[....] el temor que tenía porque la verdad es que tuve muchas crisis [...]". 
Otro sentimiento expresado por lo adultos egresados de la UCI fue la desesperación, una emoción asociada al aislamiento, soledad y miedo; la desesperación nace a partir de la incertidumbre de su situación y el temor de poder morir. Muchas veces quien se encuentra inmerso en la desesperación vive la vida de una manera angustiosa, esto lo manifestaron en los siguientes descriptores.

PE3: "[...] es caótico, desesperante [...]" "Entraba en desespero... las horas se hacian largas"

PE10: "[...] mucha incertidumbre y preocupación”.
Los participantes develaron que se imaginaban el espacio de la UCI muy distinto antes de ingresar a ella, su experiencia de hospitalización la consideraron dura y pesada. Los pensamientos de Rizzo (8) afirman que la asimilación del entorno está dada por la liberación construida contextualmente, donde el ser humano posee la capacidad

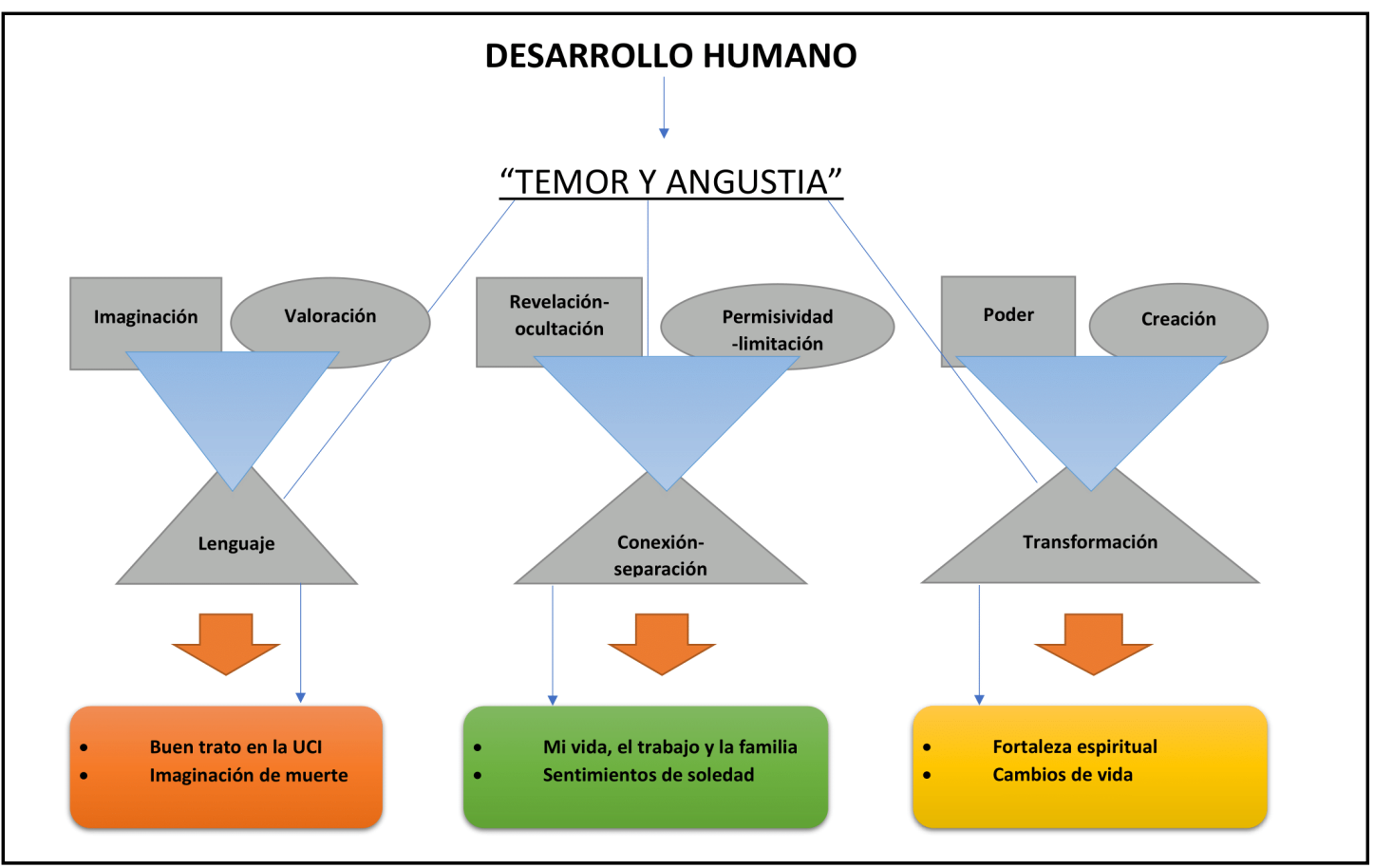

Figura 1. Esquema de temas y subtemas a la luz de la teoría del desarrollo humano de Rizzo.

Fuente: Elaboración propia de los investigadores

En esta experiencia la percepción que tiene el ser humano al recibir estímulos del exterior, lo llevan a realizar juicios sobre lo que le rodea, bien sea sitios, lugares, ruidos, sonidos entre otros. En este aspecto señalaron:

PE7: "ufff... yo me imagine que eso era algo terrible, pues uno no se la imagina diferente".

PE9: "[...] me dio duro la hospitalización, ese ambiente dia y noche, horrible [...]".

PE11: "Pesado el ambiente, muy dificil, sobre todo las luz y el ruido".

\section{de percibir e interpretar las situaciones en busca de un universo humano indivisible de cambios constantes, que unifica su experiencia a través de las relaciones con el entorno.}

1. Principio teórico: Imaginación Valoración - Lenguaje:

\section{- $\quad$ Subtema 1: Buen trato}

Los significados que construye el adulto mediante la experiencia formada por su lenguaje e imaginación, le permiten construir una realidad limitada que se expresa verbalmente a través de los valores y reflexiones presentes en una situación, por eso, enfermería como pilar de la atención provee los insumos para que el ser humano ilustre, explore e interprete sus experiencias. 
En este caso, la atención que brinda el personal de salud que labora en la UCI debe transmitir confianza y seguridad para comunicarse de manera eficaz con los pacientes. A pesar de las limitaciones y dificultades emocionales que presentan los individuos durante su hospitalización, para los adultos egresados de la UCI fue fundamental el buen trato y la atención brindada por parte del equipo interdisciplinario.

La buena atención recibida en UCI contribuye a mejores resultados en los pacientes, incluyendo reducción de la estancia, mayor satisfacción y mejor comunicación en la toma de decisiones. Esta atención también pude ser de manera indirecta en donde el paciente por medio de la tecnología recibe una vigilancia constante a través de la monitorización y sistematización. De esta manera, la atención es parte fundamental en la recuperación de los pacientes, ya que ellos sienten más apoyo y mayor seguridad durante su estancia en UCI. Los participantes expresaron los aspectos relacionados con la buena atención durante su estancia, así:

PE1: “[...] están más pendientes de uno...más monitoreado... le toman los signos vitales cada rato, están pendientes que la droga que una cosa, que la otra [...]".

PE2: [...] "yo las llamaba y enseguida estaban ahi... estaban pendiente todos".

PE6: "la atención, eso, eso fue bastante bueno, fue bueno [...], yo no tengo queja de todo el personal [....]".

PE8: "pues, muy buena la atención [...] fue súper buena, no me quejo".

\section{- Subtema 2: Imaginación de muerte}

La imaginación como principio de la teoría del desarrollo humano (12), establece la visión que tiene un individuo de la realidad, visión de su conocimiento personal y reflexivo tomado de los significados construidos en una situación inesperada, consecuente de un proceso de salud.

En el relato de la experiencia de los adultos egresado de la UCI, la percepción de gravedad y riesgo de la vida la expresaron con sensaciones de cercanía a la muerte, siendo esta una experiencia de gran impacto.

En los pacientes críticos, la idea de muerte se ve manifestada por sentimientos y temores al sufrimiento. No obstante, los participantes también refirieron reacciones emocionales en las que experimentan imaginaciones de muerte, sentimiento que se define como situación generada por cir- cunstancia en la que se encuentra un individuo al momento de verse en peligro. Los participantes refieren que:

PE3: “... siempre han dicho el que entra en uci no sale vivo [...]",

PE8:[....] "fue muy traumático... Cuando a uno le mencionan UCI... es...lo peor, el peor estado $[\ldots]$ "

PE9: "[...] nunca pensé estar hospitalizado en UCI... pensé en muchas cosas, pensé que no me iba a parar más, [...] cuando uno llegaba a una uci terminaba ...muerto [...]"

Algunos pacientes hospitalizados experimentan sentimientos de temor, incertidumbres y angustias por los sucesos de gravedad y muerte que ocurren en su estancia hospitalaria, lo cual genera una alteración en el entorno del paciente, manifestado en el siguiente descriptor:

PE2: "[...] no es nada agradable... porque uno ve muchas cosas ahi, otros se complican".

PE8:" Yo vi cuando se murió el paciente de al frente, me dio miedo, dije... yo también me iré a morir, claro uno se asusta".

\section{Principio teórico: Revelación-Ocultación, Conexión-Separación, Permisividad-Li- mitación: \\ - Subtema 3: Mi vida, el trabajo y la familia}

Los seres humanos en su cotidianidad de vida establecen pautas rítmicas que estructuran sus vivencias y experiencias; en su entorno el adulto egresado de la UCI construye patrones de relaciones con otros que provocan libertades o restricciones.

Por tanto, las limitaciones que se presentan por la hospitalización genera sentimientos de frustración, debido a las restricciones físicas, y emocionales a las que se enfrenta el enfermo por no ser independiente; así, se forjan reticencias en los aspectos familiares, sociales y laborales a causa de su enfermedad, secuelas físicas y/o emocionales.

PE3: “[...] yo estaba trabajando... tuve que renunciar... porque.... es mi vida o el trabajo $[\ldots] "$.

PE9: "[...] me toco dejar de trabajar, porque mi enfermedad me lo impedía...[...].

Otro aspecto importante que develaron los participantes fueron los sentimientos de desánimo y temor, al pensar no poder continuar la crianza de sus seres queridos, puesto que ellos eran los 
que soportaban la economía de sus hogares. Argumentan que:

PE6: "[....] mi familia es lo que más me preocupa... a mis hijos aún no he terminado de criarlos, ni educarlos, $[\ldots]$ "

PE8: "[...] soy madre cabeza de familia ... iimagínense!, yo postrada es una cama ... y era la que generaba los ingresos."

\section{- $\quad$ Subtema 4: Sentimientos de soledad}

El núcleo familiar, es considerado como uno de los aspectos más importantes para la sociedad, porque representa la principal red de apoyo, incentivo y fuente de fortaleza. De este modo, los pacientes necesitan el soporte familiar para su transformación y motivación emocional. Así, el aislamiento familiar se consideró un factor estresante que les genera angustia.

Rizzo (12), señala que las pautas de conexión y separación crean vínculos de distanciamiento entre personas cercanas, es decir, se es capaz de sobrevivir frente a la ausencia de un ser querido, pero siempre estableciendo los vínculos de conexión.

En este contexto, los participantes manifestaron sentimientos de soledad durante su estancia en la UCI, referidos como momentos en el que se sienten aislados de sus seres queridos y requerían de su cercanía como apoyo en esas situaciones difíciles a las cual se veían enfrentados:

PE1: “[...] se siente uno alejado, solo [...]"

PE9: "la primera vez si me dio duro porque [...] nunca había estado en un hospital solo".

PE10: “... me sentía solo, estaban muchos familiares... y no los dejaban entrar"

PE11: "unos quiere ver a sus hijos, esposo, a todos sus familiares, pero no los dejaban entrar"

Las restricciones de las visitas en la unidad de cuidado intensivo hacen parte de políticas institucionales y normativas que se han establecido desde su creación, sin embargo, para algunos pacientes es de suma importancia la compañía de sus familiares, y manifiestan la necesidad de sentir el apoyo como aspecto esencial a la hora de su recuperación:

PE2: "[....] falta de comunicación ... con mis familiares, ... la veía una sola horita al día”.

PE5: "[...] recibir una sola hora de visita ...uno quisiera estar más tiempo acompañados [...]”.

PE8: "[...] es dificil no recibir visitas...no pude ver a mis hijos".

\section{Principio teórico Poder - transforma- ción - creación:}

Las experiencia de enfrentarse a una situación difícil, separarse de los seres queridos e imaginar estar cerca de la muerte, despierta en el individuo sentimientos de cambio y transformación; cambios que construyen infinitas posibilidades de ser, cómo ser, y qué ser, con quien relacionarse y como transcender en el devenir visible- invisible para convertirse en el ahora emergente, en este principio se presentan dos subtemas:

\section{- Subtema 5: Fortaleza espiritual.}

Desde un punto de vista holístico, los pacientes deben satisfacer sus necesidades espirituales, en ese sentido, necesitan de su espiritualidad para mantener su fuerza interior, motivaciones y creencias, siendo estas fuentes de ayuda. Un aspecto dentro del área espiritual son las creencias religiosas, donde gran parte de los pacientes se aferran a ellas, entregan su confianza y les ayuda en la aceptación de la enfermedad, siendo una fuente de esperanza.

Los sentimientos de incertidumbre lo relacionan con el miedo, el tiempo y la vulnerabilidad por el desconocimiento del futuro inesperado. Una vez los participantes experimentan momentos de soledad por su situación clínica, sienten la necesidad de buscar un apoyo supremo, para tener tranquilidad interior y saber sobrellevar los momentos de angustia e imaginaciones de muerte, de modo que los participantes se apoyan en Dios y aumentan su fortaleza espiritual para salir adelante de esa situación, expresado así:

PE1: "espiritualmente se fortalece uno... Dios... es lo que más me fortaleció"

PE5: "[...], mi enfermedad me invito... a buscar mayor cercanía con Dios... a tener como un crecimiento espiritual [...]" "[...] todos nos aferramos a Dios, [...]"

PE8: "Dios...es el que nos da la vida, todos los días me aferraba a Dios, y le pedía por mi recuperación $[\ldots]$ "

\section{- Subtema 6: Cambios de vida.}

Los cambios hacen parte de la vida, estos son enfrentados en algún momento por el individuo, bien sea por consecuencias naturales, sociales, morales o espirituales, causando angustias y preocupaciones en el ser humano.

Cuando el ser humano descubre sus cambios mo- 
difica su comprensión, es decir, cambian las pautas de vida, la realidad del mundo y transforma profundamente el ser visible al ser ahora emergente.

Al respecto los participantes manifiestan sentir un cambio a raíz de la situación que experimentan en la UCI se presenta un aumento por el valor de la vida, son conscientes sobre las medidas de cambio que deben tomar y auto reflexionan sobre las situaciones pasadas y las situaciones presentes que favorecen al cambio.

PE1: “[...] valorar... más la vida...si va haber cambios en mi vida ... ya no va hacer igual [...]". PE10: “[...] voy a estar más cerca de mi familia y me voy apoyar en ellos".

Así mismo, los cambios involucran modificaciones importantes en el estilo de vida, cambios en la conducta y comportamientos de las personas, en ocasiones les resulta muy difícil adherirse de forma permanente al tratamiento y/o rehabilitación. Los cambios como dejar de fumar, empezar actividad física, hábitos nutricionales y en algunos casos modificar o dejar de realizar sus actividades laborales, suelen generar sentimientos de frustración e impotencia en los pacientes, pero al mismo tiempo estos cambios que les generó la estancia por la UCI, los lleva a reflexionar sobre el mantenimiento o recuperación de su salud, así como también, el significado de la familia.

PE3: "[...] sirve como experiencia... coger medidas más drásticas...de los hábitos alimenticios [...].

PE5: "[...] una situación que le permite a uno hacer un análisis global de lo que ha sido la vida... para generar grandes cambios... hacer grandes análisis a mirar que muchos hábitos que tendrá que comenzar [...]".

PE6: " uno aprender a querer más las cosas, su familia, extraña hasta su casa..."

\section{Discusión}

Los resultados hallados en este estudio resuelven una necesidad sentida de los investigadores y profesionales de enfermería donde comprender el significado de la experiencia de los pacientes que una vez pasaron por la unidad de cuidados intensivos, permitirá a todo el equipo interdisciplinario la elaboración de planes con los pacientes durante su estancia en la UCI, para que tengan un proceso de recuperación satisfactorio, lo cual mejorara su percepción y la calidad del servicio.
Cabe resaltar que los resultados encontrados coinciden con otras investigaciones que han abordado este fenómeno desde lo disciplinar como preocupación inminente y metodológico con este enfoque individualista. En lo que respecta a las características demográficas Beltrán (5), plantea en su estudio de investigación que incluyo 9 personas adultas entre los 24 y 80 años de edad que estuvieron críticamente enfermos y hospitalizados en la UCI. Igualmente, Santiago y Vargas (26), entrevisto nueve pacientes entre 59 y 74 años que estuvieron hospitalizados en una unidad de cuidado intensivo coronario con diagnóstico de infarto agudo de miocardio. Siendo consistentes con el grupo de población participante en este estudio.

La experiencia de los participantes fue descrita como "temor y angustia", los cuales son sentimientos generados al tener contacto con la unidad de cuidado intensivo, lo que es coherente con lo descrito por los autores Zambrano et al. (27), quienes refieren que los sentimientos son parte esencial de una persona en la vida cotidiana y al tener que enfrentar un proceso de enfermedad se generan en el individuo una serie de preocupaciones que pueden interferir en su recuperación. La angustia, el sufrimiento y la inestabilidad son los sentimientos que se experimentan frente a esta situación.

Así mismo Vasconelos et al. (28), en sus resultados de investigación, expresan que en algunos pacientes sobre todo en los adultos mayores, se manifiestan cambios en las relaciones familiares principalmente por el temor a la aparición de una falta importante de independencia que transforme el rol de cuidador al de sujeto cuidado.

De otra parte, Beltrán (5), concluye que los pacientes describen la experiencia y el significado que tuvo el sufrimiento el cual fue un componente de la enfermedad que contribuyó en forma importante a la dureza de la situación. La experiencia vivida por los pacientes en estado crítico en una UCI es muy compleja y llena de contrastes. Uno de esos contrastes se muestra en la manera como los participantes consideran su experiencia, que va desde "normal" e "increíble" hasta "dura", "difícil", "triste" y "horrible" para las personas que la viven.

En lo que respecta al buen trato, los participantes del estudio manifestaron la importancia de la atención por parte del personal que labora en la unidad de cuidados intensivos, Luiz, Caregnato y Costa (29), refiere que el cuidado humanizado en las UCI, hacen referencia a la capacidad de brindar la atención basada en las necesidades 
del otro, apreciando hasta lo más mínimo que incomoda o percibe el paciente como amenaza, el acompañamiento constante parte del respeto y la consideración de ese ser humano que requiere de la explicación de cada actividad a realizar para disminuir el sufrimiento y el miedo, así como también la percepción de la familia frente al cuidado.

El ingreso a la UCI generó en participantes sentimientos de temor, en donde el sentimiento de muerte fue el más manifestado según sus creencias sociales y culturales. De esta manera lo resalta Zambrano et al. (27), quienes describen que estos sentimientos son influenciados por las experiencias previas hospitalarias y por sus creencias culturales, como el temor a la muerte. Del mismo modo Blanca et al (30), concluyo que el paciente presenta miedo a la muerte cuando la siente muy cercana, desconocimiento de las consecuencias de la enfermedad y de todo lo que ocurre a su alrededor, y a un futuro imprevisto que cambie completamente su vida. Por su parte Soares, Da Cunha y Biondo (31), en coherencia con lo descrito, también manifiestan que la hospitalización en una UCI genera innumerables incertidumbres y preocupaciones, ya que es un entorno desconocido que causa temores, principalmente por la asociación con la muerte por la gravedad clínica de los pacientes hospitalizados.

Con relación al subtema Mi vida, el trabajo y la familia se tienen presentes todas aquellas frustraciones que sienten los participantes de no poder continuar con las actividades y roles que venían desempeñando antes de ingresar a la UCI y los cambios que generara dicha hospitalización. En efecto, la intervención puede presentar diversas alteraciones psicológicas como consecuencia de la adaptación a la nueva situación, en la cual los pacientes se encuentran en un ambiente extraño o desconocido, que les priva de sus interacciones y sensaciones habituales, mientras les asaltan constantemente estímulos sensoriales extraños (32).

Por este motivo, se considera que el ambiente propio de la UCI afecta psicológicamente a los pacientes de un modo negativo. Además, tienen que abandonar, al menos temporalmente, los roles que previamente desempeñaban (familia, trabajo, etc.) o incluso cambiarlos $(27-33)$.

En cuanto a los sentimientos de soledad fueron expresados por los participantes en torno a la necesidad de tener compañía por parte de sus familiares y sentir un apoyo constante de parte de ellos y de esta manera poder sentir más tranquilidad durante su hospitalización en la UCI.
Del mismo modo Beltrán (6), considera que el ambiente de soledad es percibido por los pacientes en la UCI, el hecho de que la compañía familiar estaba sometida a un esquema restrictivo y las medidas de control al ingreso de personas impuestas por la administración hospitalaria o por el equipo de salud. Agrega que la soledad se debe a la ausencia de aquellas personas cuya compañía se desea, de recibir aquellas palabras que los pacientes quieren oír, pero que no pueden escuchar de las personas que son importantes y significativas para cada uno (2). Ante esto Goularte, Gabarra, y Moré (34) expresan que los profesionales en reconocimiento de esto, perciben la flexibilización de la visita como beneficiosa para pacientes y familiares en la UCI.

Por el contrario Baharoon et al. (35), al medir la satisfacción familiar en unidades de cuidados críticos, encontraron que esta no es influenciada por la frecuencia de las visitas a pesar que reducen los síntomas de depresión y ansiedad.

La fortaleza espiritual fue considerada como la herramienta más importante que encontraron los participantes durante la estancia en la unidad de cuidado intensivo, puesto que esta los afianzó más con Dios. Refiere Collado (36), que la espiritualidad es un fenómeno íntimo y trascendente, un fenómeno vital para el desarrollo de la recuperación de la salud del paciente. También se considera una fortaleza y una fuente de motivación y búsqueda del significado de la vida. Es por esto, que el cuidado espiritual ofrecido por el personal de enfermería debe tener un sentido de responsabilidad, respeto y dedicación al paciente, sobre todo si el paciente está en el proceso de muerte. Como lo expresa Soto, Olivella y Bastidas (37), cuando el cuidado enfermero interactúa con la fragilidad y el sufrimiento del enfermo y/o la familia, lo lleva a visibilizar el cuidado espiritual, fundamentado en el respeto a la vida y a la dignidad de la persona.

Finalmente, el egreso de una unidad de cuidados intensivos va de la mano con indicaciones y prescripciones médicas, que van a orientar al paciente sobre el mejoramiento o el mantenimiento de la salud. Por tanto, los participantes del estudio reflexionan y analizan esas modificaciones que deberán tener en su vida cotidiana a raíz de la enfermedad por la cual ingresaron a la UCI. Medina et al. (38), sustentan que el diagnóstico de enfermedad crónica cambia la forma como la persona se ve a sí misma y a su vida; afecta profundamente su auto-concepto, debido a que el envejecimiento y las modificaciones de la esfera psicológica dificultan la adaptación a la enfer- 
medad, su proyecto de vida deberá ser revisado y a menudo, no podrá saber cuál será su curso. Así mismo Baeza y Quispe (39), develan que el síndrome post-cuidados intensivos (PICS) afecta entre el $30 \%$ y el $50 \%$ de pacientes después de la enfermedad crítica y se caracteriza por síntomas físicos, neuropsicológicos o emocionales tales como ansiedad, depresión o estrés post- traumático; estos problemas empiezan a aparecer cuando el paciente está en la UCI y pueden persistir después de su de alta, sus consecuencias impactan en la calidad de vida de los pacientes y sus familias.

Por último, Díaz y Mercado (40), describen que el comportamiento y los modelos de afrontamiento de las personas a la enfermedad pueden desempeñar un papel importante en su curso y en el hecho de que un paciente cumpla o no con las prescripciones médicas 1 . Podrán alcanzar un mejor control de la enfermedad e incrementar o perseverar su calidad de vida, aquellas personas que logren adherirse adecuadamente a los regímenes conductuales que cada una de las enfermedades exige para lograr una buena evolución; además de considerar que los profesionales de salud realicen, junto con las personas enfermas y sus familias, una planificación anticipada de los cuidados, ya que se ha reportado que ello beneficia a los pacientes.

En general, los hallazgos proporcionan un soporte teórico a la investigación en enfermería para identificar los fenómenos, que aunque no son medibles si pueden ser comprendidos, lo que permitirá un análisis global y holístico de los pacientes y las diversas circunstancias que viven por algún deterioro de su salud sea físico o psicológico en donde se ve inmersa la familia y contribuir de una manera muy creativa en la resolución de problemas identificados.

\section{Conclusiones}

- De acuerdo a los hallazgos se concluye que la estancia de los pacientes adultos en la unidad de cuidado intensivos, desencadeno experien- cias y sentimientos generados por factores como el desconocimiento del entorno, percepciones sociales y culturales sobre la muerte, además de cambios como aprender a valorar a la familia y aprovechar el tiempo junto a ella.

- Al comprender la experiencia sentida por los participantes se puede afirmar, que para ellos es primordial el apoyo del núcleo familiar, el buen trato por parte del equipo interdisciplinario y aprender el valor de la familia y el tiempo vivido.

- La unidad de cuidado intensivo cuenta con unas restricciones de visitas las cuales son protocolarias e institucionales, este tipo de hospitalización al ser distinta para ellos, les generó la necesidad de tener a sus familiares cerca en el proceso de recuperación; lo que de cierta forma desencadenó en ellos aparte del temor y angustia, el sentimiento de soledad por sentirse aislados y dependientes de otras personas; buscando así un mayor afianzamiento espiritual y cercanía con Dios.

- La buena atención por el equipo interdisciplinario de la UCI, logró establecer en los pacientes seguridad y tranquilidad durante su estancia, dichas acciones permitieron minimizar sentimientos negativos, logrando de esta manera una recuperación más satisfactoria en proceso de la hospitalización, destacando la importancia de la humanización de los cuidados.

- Por último, la unidad de cuidado intensivo originó cambios y transformaciones en la vida de los participantes, muchos de ellos en consecuencia por su patología, lo que afecto algunos los roles laborales y sociales que desempeñaban.

\section{Conflicto de Intereses}

Los autores declaran no tener ningún conflicto de intereses.

\section{Referencias Bibliográficas}

1. Santiago de Castro E, Vargas-Rosero E. Experiencia de estar hospitalizado en una unidad de cuidado intensivo coronario de Barranquilla. Av. Enferm [Internet]. 2015 [Citado 04/09/17];33(3):381390. Disponible en: http://www.scielo.org.co/pdf/aven/v33n3/v33n3a06.pdf

2. Soto del Arco F. Manual de ventilación mecánica para enfermería. 1ra ed. Editorial Médica Panamericana.; 2017. 
3. Bohórquez A, Ramírez AC, Rodríguez EE, Polentino CV, Aceros MZ. Suspiro de vida: vivencias de los pacientes de la Unidad de Cuidados Intensivos sometidos a soporte ventilatorio. Rev. cienc. cuidad. [Internet]. 2016 [citado 16 de mayo de 2020];8(1):61-9. Disponible en: https://revistas. ufps.edu.co/index.php/cienciaycuidado/article/view/482

4. Amorós-Cerdá S, Arévalo-Rubert, MJ, Maqueda-Palau R, Pérez-Juan E. Percepción de la intimidad en pacientes hospitalizados en una Unidad de Cuidados Intensivos. Enf intensiva [Internet]. 2008 [ consultado 16 de octubre de 2018];19(4):1-11. Disponible en: https://dialnet.unirioja.es/ servlet/articulo? codigo $=2783407$

5. Beltrán-Salazar O. La experiencia de estar hospitalizado en una unidad de cuidado intensivo. Rev. Aquichan [Internet]. 2009 [ consultado 30 de abril de 2018] ;9(1):1-9. Disponible en: https:// aquichan.unisabana.edu.co/index.php/aquichan/article/view/167/1668

6. Beltrán-Salazar O. Estar críticamente enfermo significa no ser capaz y no poder decidir. Index Enferm [Internet]. 2008. [ consultado 30 de abril de 2018]; 17(2):92-96. Disponible en: http:// scielo.isciii.es/scielo.php?script=sci_arttext\&pid=S1132-12962008000200003

7. Prieto-Martínez P, Suárez Rodríguez N, Gómez Gómez L, Cárdenas Bolivar Y, Celis-Rodriguez É. Percepciones de dignidad y respeto en Unidades de Cuidado Intensivo. Acta Colombiana de Cuidado Intensivo. [Internet]. 2020 [consultado 16 de mayo de 2020]; 20(1):1-74. Disponible en: https://doi.org/10.1016/j.acci.2020.01.005

8. Mijangos-Fuentes K. El Paradigma Holístico de la Enfermería. Rev Salud y Adm. [Internet]. 2014 [consultado 9 de mayo de 2019]; 1(2):1-6. Disponible en: https://revista.unsis.edu.mx/index. $\mathrm{php} / \mathrm{saludyadmon/article/view/86}$

9. Peña-Ortiz LM, Hernández-Moncada YA, Morales-Morales JM, Zambrano-Plata G, Sánchez C. Life heartbeat: experiences of patients with cardiovascular disease in an intensive care unit. Rev. cienc. cuidad. [Internet]. 2019 [citado 14 de mayo de 2020]; 16(2):46-8. Disponible en: https://revistas.ufps.edu.co/index.php/cienciaycuidado/article/view/1607

10. Martínez-González L, Olvera-Villanueva G. El paradigma de la transformación en el actuar de enfermería. Rev Enferm IMSS [Internet]. 2011 [consultado 30 de abril de 2018]; 19(2):105-7. Disponible en: https://www.medigraphic.com/cgi-bin/new/resumen.cgi?IDARTICULO $=31760$

11. Baeza-Gómez I, Quispe-Hoxsas L. Proyecto "Humanizando los Cuidados Intensivos", nuevo paradigma de orientación de los Cuidados Intensivos. Rev. Bioética y Derecho [Internet]. 2020 [citado 2020 mayo 16]; (48):111-126. Disponible en: http://scielo.isciii.es/scielo.php?script=sci_arttext\&pid $=$ S1886-58872020000100008\&lng $=$ es

12. Alligood-Raile M. Desarrollo humano. In: Modelos y teorías en enfermería. OCTAVA. Barcelona España; 2015. p. 451-81.

13. Heidegger M. Ser y tiempo. Madrid: Trota; 2012.

14. Mendieta-Izquierdo G, Ramírez-Rodríguez J, Fuerte J. La fenomenología desde la perspectiva hermenéutica de Heidegger: una propuesta metodológica para la salud pública. Revista Facultad Nacional de Salud Pública: El escenario para la salud pública desde la ciencia [Internet]. 2015 [consultado 8 de septiembre de 2019]; 33(3):435-443. Disponible en: https://dialnet.unirioja.es/servlet/ articulo? codigo $=5214008$

15. Muñoz-Pérez E. Trascendencia, mundo y libertad en el entorno de Ser y Tiempo de Martín Heidegger. Veritas [Internet]. 2015 [consultado 8 de septiembre de 2019]; 32:95-110. Disponible en: https://scielo.conicyt.cl/scielo.php?pid=S0718-92732015000100005\&script=sci_arttext

16. Castillo-Espitia E. La fenomenología interpretativa como alternativa apropiada para estudiar los fenómenos humanos. Investigación y Educación en Enfermería [Internet]. 2000 [consultado 8 de septiembre de 2019]; 18(1):27-35. Disponible en: http://portal.amelica.org/ameli/jatsRepo/105218294002

17. Denzin NK, Lincoln S. Collecting an interpreting qualitative materials. London: Sage Publications.; 1998. p.179-210. 
Keydis Sulay Ruidiaz-Gómez, Shirley Fernández-Aragón.

18. Creswell JW. Qualitative inquiry and research design: choosing among five traditions. London: Thousand Oaks, CA. Sage; 1997.

ISSN-PRINT

$1794-9831$

E-ISSN 2322-7028

Vol. 17 No. 3

Sep - Dic 2020

Cúcuta, Colombia

19. Starks H, Brown-Trinidad S. Choose your method: a comparison of phenomenology, discourse analysis, and grounded theory. Qualitative health research [Internet]. 2007 [consultado 16 de Octubre de 2018]; 17(10):1372-1380. Disponible en: https://pdfs.semanticscholar.org/a317/738b3a28d7c2c1af03fd1e22f67058342781.pdf

20. Lincoln YS, Guba EG. Naturalistic Inquiry. Estados Unidos: Age Publications.;1985.

21. Guba EG, Lincoln YS. Epistemological and methodological bases of naturalistic inquiry. Educ Tech Res Dev [Internet]. 1982 [consultado 16 de octubre de 2018]; 30(4):233-52. Disponible en: https://link.springer.com/article/10.1007/BF02765185

22. Bonilla-Castro E, Rodríguez-Sehk P. Más allá del dilema de los métodos: la investigación en ciencias sociales. Buenos Aires.: Editorial Norma.; 2005.

23. Critical Appraisal Skills Programme Español. Entendiendo la evidencia de estudios cualitativos 10 preguntas para ayudarte a entender un estudio cualitativo. [Internet]. 2016 [citado el 25 de mayo de 2019]. p. 1-6. Disponible en: http://www.redcaspe.org/herramientas/instrumentos.

24. República de Colombia. Ministerio de la Protección Social. Resolución No. 8430, octubre 4, por la cual se establecen las normas científicas, técnicas y administrativas para la investigación en salud. [Internet]. Bogotá D.C.: Ministerio de la Protección Social; 1993 [consultado el 10 de noviembre de 2015]. Disponible en: https://www.minsalud.gov.co/sites/rid/Lists/BibliotecaDigital/RIDE/DE/ DIJ/RESOLUCION-8430-DE-1993.PDF

25. Pellegrini A, Macklin R. Investigación en Sujetos Humanos: Experiencia Internacional. Santiago de Chile: Programa Regional de Bioética OPS/OMS; 1999: 33-46.

26. Santiago-de-Castro E, Vargas-Rosero E. Experiencia de estar hospitalizado en una unidad de cuidado intensivo coronario de Barranquilla. Av.enferm. [Internet]. 2015 [Consultado 15 febrero 2018]; 33(3):381-90. Disponible en: http://bdigital.unal.edu.co/60480/1/41841-281572-1-PB.pdf

27. Zambrano-Plata G, Ferreira-Cardona J, Lindarte-Clavijo A, Niño-Bayona C, Ramírez-Rodríguez N, Rojas-Bautista L. Entre la incertidumbre y la esperanza: Percepciones y motivaciones de los pacientes hospitalizados en la unidad de cuidados intensivos (UCI). Rev. cienc. cuidad. [Internet]. 2016 [Consultado 15 Febrero 2018]; 7(1):52-60 Disponible en: http://revistas.ufps.edu.co/ojs/index. $\mathrm{php} /$ cienciaycuidado/article/view/493

28. Vasconelos EV, Freitas KO, Silva SED, Baia-RSM, Tavares RS, Araújo JS. O cotidiano de familiares de pacientes interna-dos na uti: um estudo com as representações sociais. Re-vista de Pesquisa Cuidado é Fundamental [Internet]. 2016 [acceso 16 may 2020]; 8(2):4313-4327. Disponível em: http://www.seer.unirio.br/index.php/cuidadofundamental/article/view/4366

29. Luiz FF, Caregnato RCA, Costa MR. Humanization in the Intensive Care: perception of family and healthcare professionals. Revista brasileira de enfermagem [Internet]. 2017 [acceso 16 may 2020]; 70(5):1040-1047. Disponível em: http://www.scielo.br/scielo.php?script=sci_arttext\&pi-d=S0034-71672017000501040

30. Blanca-Gutiérrez JJ, Blanco-Alvariño A, Luque-Pérez M, Ramírez-Pérez M. Experiencias, Percepciones y Necesidades En La Uci: Revisión Sistemática De Estudios Cualitativos. Enferm glob. [Internet]. 2008 [Consultado 15 febrero 2018]; 12(1):1-14. Disponible en: https://revistas.um.es/ eglobal/article/view/822/842

31. Chaves-Soares E, Pinheiro da Cunha JX, Santana-Biondo C, Lomanto-Rocha AK. Representação social de pacientes sobre a unidade de terapia intensiva. Revista Enfermagem Atual In Derme, [Internet]. 2020 [Consultado 6 Mayo de 2020]; 91(29):33-39 Disponible en: http://revistaenfermagematual.com/index.php/revista/article/view/531/602

32. Amengual G. El concepto de experiencia: de Kant a Hegel. Topicos [Internet]. 2007 [Consultado 15 febrero 2018]; 15:1-20. Disponible en: https://www.redalyc.org/pdf/288/28811907001.pdf

33. Cabrera-Saavedra J, Soledad-Rivera M, Jaman-Mewes P. Significados de la experiencia de 
una enfermera con su madre hospitalizada en una unidad intensiva coronaria. Revista Horizonte de Enfermería [En línea]. 2020 [Consultado 15 Mayo 2020]; 25(1):23-31. Disponible en: https://doi. org/10.7764/Horiz Enferm.25.1.23

34. Nunes-Goularte P, Macedo-Gabarra L, Ojeda-Ocampo CL. A visita em Unidade de Terapia Intensiva adulto: perspectiva da equipe multiprofissional. Rev. Psicol. Saúde [Internet]. 2020 Abr [Consultado 6 Mayo de 2020]; 12(1): 157-170. Disponible en: http://pepsic.bvsalud.org/scielo. php?script $=$ sci_arttext\&pid $=$ S2177-093X2020000100012\&lng=pt.

35. Baharoon S, Al-Yafi W, Al-Qurashi A. Al-Jahdali H, Tamim H, Alsafi E, Al-Sayyari AA. Family Satisfaction in Critical Care Units: Does an Open Visiting Hours Policy Have an Impact?, Journal of Patient Safety. [Internet]. 2017 [Consultado 16 Mayo de 2020]; 13(3): 169-174. Disponible en: https://www.doi.org/10.1097/PTS.0000000000000140

36. Collado R. Cuidado espiritual, labor del profesional de Enfermería. Rev 360 [Internet]. 2010 [Consultado 2 dic 2018]; 5:1-3 Disponible en: http://cremc.ponce.inter.edu/360/revista360/ciencia/ Cuidado\%20espiritual\%20enfermeria.pdf

37. Soto-Morales AM, Olivella-Fernandez MC, Bastidas-Sanchez CV. Cuidado espiritual al adulto mayor, elemento del conocimiento y práctica de enfermería. Rev. cienc. cuidad. [Internet]. 2020 [citado 16 de mayo de 2020]; 17(1):123-31. Disponible en: https://revistas.ufps.edu.co/index. php/cienciaycuidado/article/view/1563

38. Medina-Fernández I, Polanco-Tinal P, Tello-García M, Esparza-González S. Propuesta de cuidados de enfermería en la adaptación de autoconcepto de adultos mayores con diabetes tipo 2. Revista Española de Enfermería de Salud Mental [Internet]. 2019 [Consultado 9 septiembre de 2019]; 6:27-32. Disponible en: http://www.reesme.com/index.php/REESM-AEESME/article/view/28/36

39. Baeza-Gómez I, Quispe Hoxsas L. Proyecto "Humanizando los Cuidados Intensivos", nuevo paradigma de orientación de los Cuidados Intensivos. Revista de Bioética y Derecho, [Internet]. 2020. [Consultado 7 Mayo de 2020] 48:111-126. Disponible en: https://revistes.ub.edu/index.php/ RBD/article/view/29173

40. Díaz-Medina BA, Mercado-Martínez FJ. Obstáculos y estrategias de afrontamiento en la atención renal: estudio cualitativo en jóvenes con enfermedad renal crónica en diálisis peritoneal. Saude soc. [Internet]. 2019 [Consultado 9 septiembre de 2019]; 28(1):275-286. Disponible en: https://www.scielosp.org/pdf/sausoc/2019.v28n1/275-286/es 